\title{
Angiotensin-Converting Enzyme 2 Deficiency Aggravates Glucose Intolerance via Impairment of Islet Microvascular Density in Mice with High-Fat Diet
}

\author{
Li Yuan, Ying Wang, Chunli Lu, and Xiaoya Li \\ Department of Endocrinology, Union Hospital, Tongji Medical College of HuaZhong, University of Science \& Technology, \\ Wuhan 430022, China \\ Correspondence should be addressed to Li Yuan; yuanli18cn@yahoo.com.cn
}

Received 15 January 2013; Accepted 20 February 2013

Academic Editor: Raffaele Marfella

Copyright (C) 2013 Li Yuan et al. This is an open access article distributed under the Creative Commons Attribution License, which permits unrestricted use, distribution, and reproduction in any medium, provided the original work is properly cited.

\begin{abstract}
The aim of this study was to evaluate the effects of angiotensin-converting enzyme 2 (ACE2) on glucose homeostasis and islet function in mice. Male wildtype (WT) and ACE2 knockout (ACE2 KO) mice were divided into chow diet group and long-term high-fat diet (HFD) group. After 16 weeks of feeding, the islet function of the animals was evaluated by intraperitoneal glucose tolerance test (IPGTT) and intraperitoneal insulin releasing test (IPIRT). The pancreas was immunohistochemically stained to analyze the relative content of insulin (IRC), vascular endothelial growth factor (VEGF), and microvessel density (MVD) in islets. There was no difference of body weight, area under curve of glucose (AUCG), area under curve of insulin from 0 to 5 min (AUGI m-5 ), MVD, and RVC (relative content of VEGF) between WT and ACE2 KO mice with regular chow diet. Under the condition of long-term HFD, the AUCG of ACE2 KO mice was increased obviously in comparison with the WT mice, with decreased IRC, MVD, AUGI ${ }_{0-5}$, $\mathrm{AUCI}_{0-30}$, and RVC (all $P<0.05$ ). In conclusion, these results show that ACE2 deficiency deteriorates islet function of mice with long-term HFD via impairment of islet microvasculature.
\end{abstract}

\section{Introduction}

The classical renin-angiotensin system (RAS), ACE-Ang IIAT1 receptor axis, is a coordinated hormonal cascade facilitating the dynamic control of perfusion in both health and disease [1]. In 2000, a new member of the RAS, ACE2, was discovered by two independent groups [2,3]. ACE2 is capable of metabolizing Ang II to generate Ang (1-7) [4]. Ang (1-7) interacting with its receptor Mas elicits numerous actions that counterbalance those of Ang II [5]. Accumulating evidence have indicated that ACE2-Ang (1-7)-Mas axis acts as a negative regulator of the ACE-Ang II-AT1 receptor axis [6].

During the past decades, the existence of a local RAS has been confirmed in both the endocrine and exocrine pancreas [7-9]. In 1991, an intrinsic RAS in the pancreas was firstly described by Chappell et al. [10]. Lau et al. [11] identified the expression of several RAS components in mouse pancreatic islet, such as angiotensinogen, ACE, AT1, and AT2 receptors. The evidence for the existence of these above components were also observed in human pancreas [12]. In addition,
ACE2 mRNA and protein have been identified in the pancreatic islets [13]. Canine pancreatic Ang (1-7) [10] and rat pancreatic Mas receptor have also been confirmed [14].

Previous studies $[15,16]$ have provided additional evidence for the close association of RAS with pancreatic endocrine physiology and its pathophysiology. Islet angiotensinogen and AT1 receptor expression were increased in the Zucker diabetic fatty rats [17]. Islet RAS activation is responsible for the islet dysfunction through exacerbation of impairment of islet blood flow [8], promoting islet cell destruction, fibrosis, and apoptosis [18]. Treatment with ACEI or ARB ameliorates the impairment of glucose tolerance [19], reduces new-onset diabetes [20], and prevents the development of diabetic complications [21]. To date, there have been limited studies on whether ACE2 plays a key role in islet function. Wong et al. [22] reported that there was no difference between the plasma glucose concentrations in the ACE2 KO mice and WT mice. The exact functions of ACE2 in the regulation of islet function remain controversial and as yet inadequately elucidated. Thus, the present study aimed to investigate the potential role 
of ACE2 in glucose homeostasis and explore the mechanisms of ACE2 in islet function.

\section{Materials and Methods}

2.1. Animals. Male ACE2 KO mice and their age matched male wildtype littermates $(\mathrm{C} 57 \mathrm{BL} / 6 \mathrm{~J})$ at the age of 5 weeks were purchased from the Institute of Laboratory Animal Science, Chinese Academy of Medical Sciences. ACE2 KO mice were maintained on the C57BL/6J background. Mice were individually housed under specific pathogen-free conditions with a 12 hours light-dark cycle, ambient temperature of $22^{\circ} \mathrm{C}$, and allowed free access to food and water. After 2 weeks of acclimatization, the mice were randomly divided into two groups. Groups of mice were fed regular rodent chow diet ( $20 \%$ protein, $70 \%$ carbohydrate, and $10 \%$ fat) or high-fat diet (20\% protein, $20 \%$ carbohydrate, and $60 \%$ fat) for 16 weeks. The body weight of each mice was measured weekly during the study. The institutional animal care and use committee approved all procedures.

2.2. Intraperitoneal Glucose Tolerance Test (IPGTT). Mice were fasted for 6 hours and were injected intraperitoneally with glucose at $2.0 \mathrm{~g} / \mathrm{kg}$ body weight. Blood was collected from the tail. Blood glucose was analyzed at $0,15,30,60$, and $120 \mathrm{~min}$ after the injection. The values of AUC were calculated. The area under the curve (AUC) was calculated using the formula $\mathrm{AUCG}=7.5 \mathrm{G}_{0}+15 \mathrm{G}_{15}+22.5 \mathrm{G}_{30}+45 \mathrm{G}_{60}+$ $30 G_{120}$ where $G_{0}, G_{15}, G_{30}, G_{60}$, and $G_{120}$ were blood glucose level at each time point during IPGTT. Blood glucose was measured using an automatic glucometer (One Touch, Byer).

2.3. Intraperitoneal Insulin Releasing Test (IPIRT). For insulin secretion test, mice were fasted for 6 hours, and glucose $(3.0 \mathrm{~g} / \mathrm{kg}$ body weight) was injected intraperitoneally. Blood samples were collected from the orbital venous plexus at 0,2 , 5,15 , and $30 \mathrm{~min}$. Insulin AUC was calculated by the formula $\mathrm{AUCI}_{0-30}=\mathrm{I}_{0}+2.5 \mathrm{I}_{2}+6.5 \mathrm{I}_{5}+12.5 \mathrm{I}_{15}+7.5 \mathrm{I}_{30}$. The AUCI from $0 \mathrm{~min}$ to $5 \mathrm{~min}\left(\mathrm{AUCI}_{0-5}\right)$ which represented the first stage insulin secretion was calculated as follows: $\mathrm{AUCI}_{0-5}=\mathrm{I}_{0}+$ $2.5 \mathrm{I}_{2}+1.5 \mathrm{I}_{5} \cdot \mathrm{I}_{0}, \mathrm{I}_{2}, \mathrm{I}_{5}, \mathrm{I}_{15}$, and $\mathrm{I}_{30}$ represented the insulin level at $0,2,5,15,30 \mathrm{~min}$ respectively. Insulin levels were measured with the mouse insulin enzyme-linked immunosorbent assay kit.

2.4. Insulin Tolerance Test (ITTs). For insulin tolerance test, mice were fasted for 6 hours and then injected insulin ( $0.5 \mathrm{U} / \mathrm{kg}$ body weight) (Novolog) intraperitoneally. Glucose levels were measured at $0,15,30,60$, and $90 \mathrm{~min}$ after the injection. Results are shown as percentage of glucose levels at the time of injection.

2.5. Islet Immunohistochemistry. Pancreas specimens were fixed in $4 \%$ chilled paraformaldehyde and embedded in paraffin. Sections were mounted on glass slides and processed for immunohistochemistry staining. The pancreatic islets were stained for insulin, CD31, and vascular endothelial growth factor (VEGF) using anti-rat primary antibody with suitable concentration. The slides were then incubated overnight at $4^{\circ} \mathrm{C}$. After three rinses with $\mathrm{PBS}$, a secondary antibody (Sigma) was applied for $30 \mathrm{~min}$ at $37^{\circ} \mathrm{C}$. A third antibody (Sigma) was applied as the same way, and $\mathrm{DAB} / \mathrm{H}_{2} \mathrm{O}_{2}$ staining was used at last. Five discontinuous sections from each animal were stained and each section was analyzed randomly and double-blindly, using light microscopy, the results were captured and evaluated by a computer image analysis system (Motic, Wetzlar, Germany).

2.6. Islet Morphology and Insulin Expression. Insulin staining was used to assess the change of islets morphology and insulin concentration in $\beta$ cells. The reciprocal of gray scale of intraislet insulin-positive staining was computed, the natural logarithm was calculated as intraislet insulin relative concentration (IRC), which represented the insulin reserve in endochylema of $\beta$ cells. Insulin-positive cell density (ICD), which was the ratio of intraislet insulin-positive nuclear quantity to insulin-positive staining area, was used to reflect the amount of beta cells intraislets.

2.7. Islet Microvascular Vessel Density (MVD). The MVD of islets was calculated by CD31-positive cells as reported [23]. Any brown-stained endothelial cell or endothelial cell cluster in islets was regarded as a microvessel for counting. Six discontinuous sections from each animal were stained and 8-10 islets in each section were selected randomly. The percentage of the area for CD31 positive cell was counted divided by the islet area to obtain MVD. All counts were performed by two investigators simultaneously and independently. The reciprocal of gray scales of intraislet VEGF positive staining was computed, and the natural logarithm was calculated to present the relative content of vascular endothelial growth factor (VRC).

2.8. Statistical Analysis. All statistical analyses were performed using SPSS 11.5. Data are expressed as mean \pm SEM. Statistical significance of differences was assessed using oneway analysis of variance, and unpaired Student's $t$-test, as appropriate. A $P$ value $<0.05$ was considered significant.

\section{Results}

3.1. Body Weight. The body weight of mice was shown in Figure 1. There was no obvious difference in body weight among the four groups at the beginning of the study. The body weights of WT and $\mathrm{KO}$ (ACE2 KO) groups were similar at end of the study. When fed with high-fat diet, the body weights of WH (WT with high-fat diet) and KH (ACE2 KO with high-fat diet) groups gradually increased. After 16 weeks high-fat diet, the body weights of $\mathrm{WH}$ and $\mathrm{KH}$ groups were significantly higher than those in corresponding chow diet groups (both $P<0.05$ ). When the study was completed, no significant difference of body weight was observed in $\mathrm{KH}$ group as compared with those in WH group (Table 1).

3.2. Islet Function. The islets function was evaluated by intraperitoneal glucose tolerance test (IPGTT) and intraperitoneal insulin releasing test (IPIRT). Fasting blood glucose levels were not significantly different among those four groups. In 
TABLE 1: Body weight, blood glucose, blood insulin, and islet function (mean \pm SD).

\begin{tabular}{lcccc}
\hline & WT & WH & KO & KH \\
\hline Body weight $(\mathrm{g})$ (beginning) & $22.60 \pm 0.65$ & $22.73 \pm 0.55$ & $22.48 \pm 0.33$ & $22.56 \pm 0.33$ \\
Body weight $(\mathrm{g})($ ending) & $29.05 \pm 0.83$ & $34.24 \pm 1.45^{*}$ & $29.21 \pm 0.69$ & $34.41 \pm 0.69^{* \#}$ \\
Fasting blood glucose $(\mathrm{mmol} / \mathrm{L})$ & $8.18 \pm 0.72$ & $8.38 \pm 0.42$ & $8.60 \pm 0.46$ & $8.63 \pm 0.55$ \\
Fasting blood insulin $(\mathrm{ng} / \mathrm{mL})$ & $0.84 \pm 0.02$ & $0.97 \pm 0.02^{*}$ & $0.87 \pm 0.01$ & $0.85 \pm 0.03^{\triangle}$ \\
AUCI0-5 $((\mathrm{ng} / \mathrm{mL}) * \mathrm{~min})$ & $9.65 \pm 0.13$ & $9.31 \pm 0.06^{*}$ & $9.58 \pm 0.15$ & $7.40 \pm 0.15^{* \# \Delta}$ \\
AUCI0-30 $((\mathrm{ng} / \mathrm{mL}) * \mathrm{~min})$ & $43.82 \pm 0.21$ & $61.61 \pm 0.56^{*}$ & $44.31 \pm 0.31$ & $44.93 \pm 0.71^{\triangle}$ \\
\hline
\end{tabular}

WT: wild type mice; WH: wild type mice with high-fat diet; KO: ACE2 knockout mice; KH: ACE2 knockout mice with high-fat diet, versus WT ${ }^{*} P<0.05$, versus $\mathrm{KO}^{\#} P<0.05$, and versus $\mathrm{WH}^{\triangle} P<0.05$.

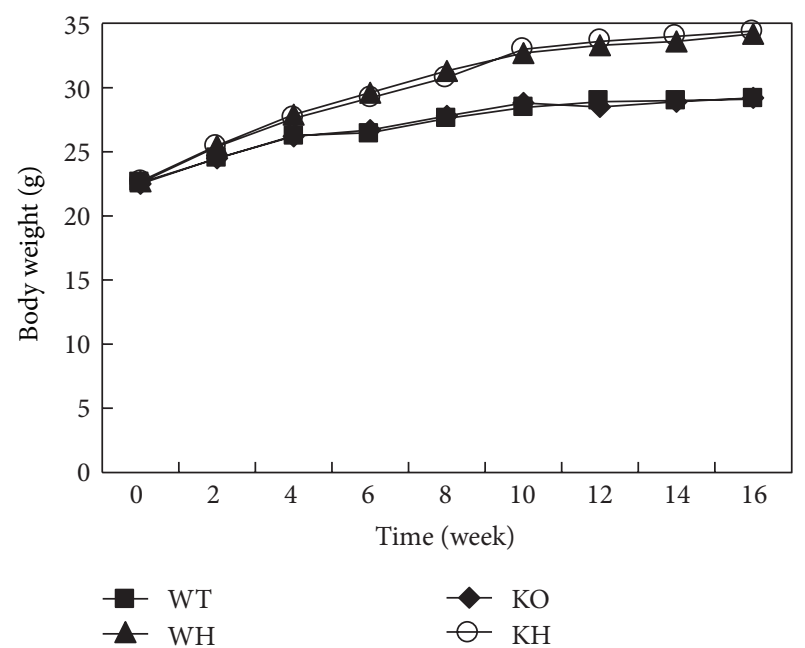

FIGURE 1: The curve of body weight. WT: wildtype mice; WH: wildtype mice with high-fat diet; KO: ACE2 knockout mice; $\mathrm{KH}$ : ACE2 KO mice with high-fat diet.

IPGTT, blood glucose level in WT group peaked at approximately $22.9 \mathrm{mmol} / \mathrm{L}$ and approached baseline levels by $120 \mathrm{~min}$. Similar glucose profiles were observed in $\mathrm{KO}$ in response to glucose challenge. The values of 30 and $60 \mathrm{~min}$ glucose of WH group were significantly higher than those in WT group (both $P<0.05$ ). However, blood glucose concentration in $\mathrm{KH}$ group peaked at approximately $30.9 \mathrm{mmol} / \mathrm{L}$ and remained elevated. We took the area under the IPGTT curve (AUCG) to quantify glucose tolerance. There was no significant difference of AUCG between WT mice and KO mice. The AUCG in WH mice was significantly elevated than that in WT mice $(P<0.05)$. The AUCG in KH groups was obviously increased than that in $\mathrm{KO}(P<0.05)$. Interestingly, we observed increased AUCG in KH mice compared with WH mice $(P<0.05)$. These data indicated that deletion of ACE2 leads to more severe glucose intolerance induced by high-fat diet (Figure 2, Table 1).

The fating blood insulin between WT group and $\mathrm{KO}$ group was not different. However, the fasting blood insulin levels in WH group were significantly higher than those in WT and $\mathrm{KH}$ groups (both, $P<0.05$ ). In the IPIRT, insulin peak climbed rapidly to about 4 -fold as basic level in $2 \mathrm{~min}$ in WT group after glucose challenge. The levels of insulin release in the $\mathrm{KO}$ group stimulated by glucose infusion were almost the same as those in the WT group. The $\mathrm{AUCI}_{0-30}$ and $\mathrm{AUCI}_{0-5}$ were not different between WT and $\mathrm{KO}$ group. Compared with WT group, the insulin secretion of $\mathrm{WH}$ group increased slowly with a delayed and increased peak. We observed a decreased $\mathrm{AUCI}_{0-5}$ and increased $\mathrm{AUCI}_{0-30}$ in WH group compared with those in WT group (both $P<0.05)$, indicating the impairment of first stage insulin releasing and a compensatory enhancement of insulin release under the condition of high-fat diet. The $\mathrm{AUCI}_{0-30}$ in $\mathrm{KH}$ group was not increased when compared with that in $\mathrm{KO}$ group. In addition, the insulin secretion in $\mathrm{KH}$ and $\mathrm{WH}$ group climbed to the peak at the same time, whereas, the former exhibited a lower peak releasing after glucose load. This finding suggested that ACE2 deficiency inhibits the compensatory enhancement of insulin secretion in response to the increased metabolic demand (Figure 3, Table 1).

3.3. Insulin Tolerance Test. In IPITT, blood glucose level in WT group dropped obviously and approached bottom levels by $30 \mathrm{~min}$. Similar glucose disappearance rate was observed in $\mathrm{KO}$ mice. After an intraperitoneal injection of insulin, the glucose disappearance rate of 15 and $30 \mathrm{~min}$ was higher in $\mathrm{WH}$ and $\mathrm{KH}$ mice than those in corresponding mice with chow diet (both $P<0.05$ ). There was no difference of the glucose disappearance rate between $\mathrm{KH}$ and $\mathrm{WH}$ mice (Figure 4).

3.4. IRC and ICD of Islets. Islets in WT group were regular with high expression of insulin. There was no significant difference of IRC and ICD between WT and KO groups. The islet area in the pancreases of WH group was larger than that in WT group, and the IRC dropped obviously $(P<0.05)$, indicating the reduction of insulin reserve in WH group. In comparison with $\mathrm{KO}$ mice, $\mathrm{KH}$ mice exhibited a decreased IRC $(P<0.05)$. Moreover, a decreased IRC was noted in $\mathrm{KH}$ group in comparison with WH group $(P<0.05)$, implying more severe impairment of insulin reserve in $\mathrm{KH}$ group. The ICD in the WH group was lower than that in the WT group, but the difference was not significant. No difference of ICD was observed between $\mathrm{KH}$ group and $\mathrm{KO}$ group. There was no difference of ICD between $\mathrm{KH}$ group and $\mathrm{WH}$ group (Figure 5, Table 2).

3.5. MVD in Islets. The MVD of islet was slightly decreased in KO mice than that in WT mice, but the difference was not significant. Staining of the pancreas using anti-CD31 antibody 


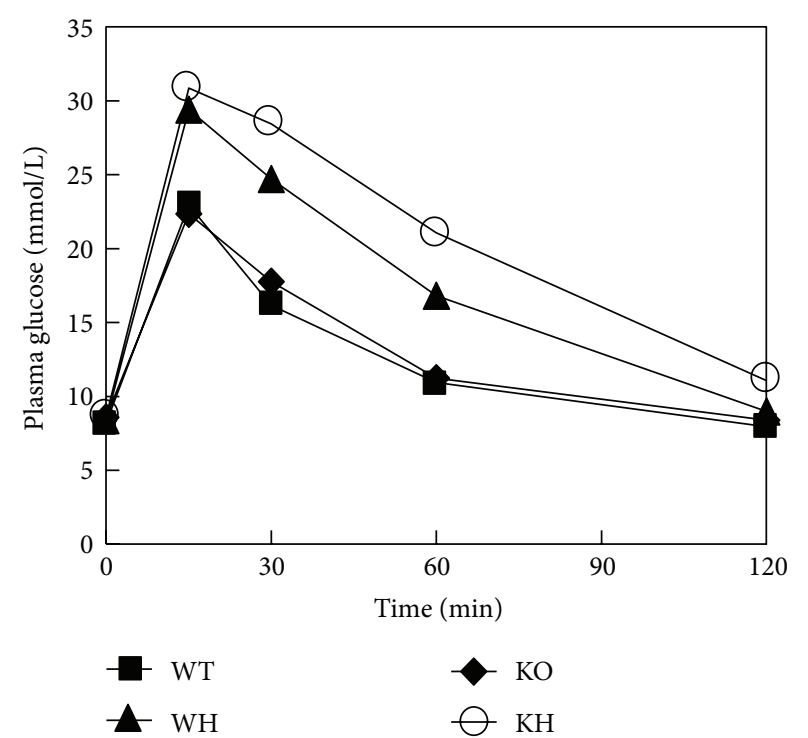

(a)

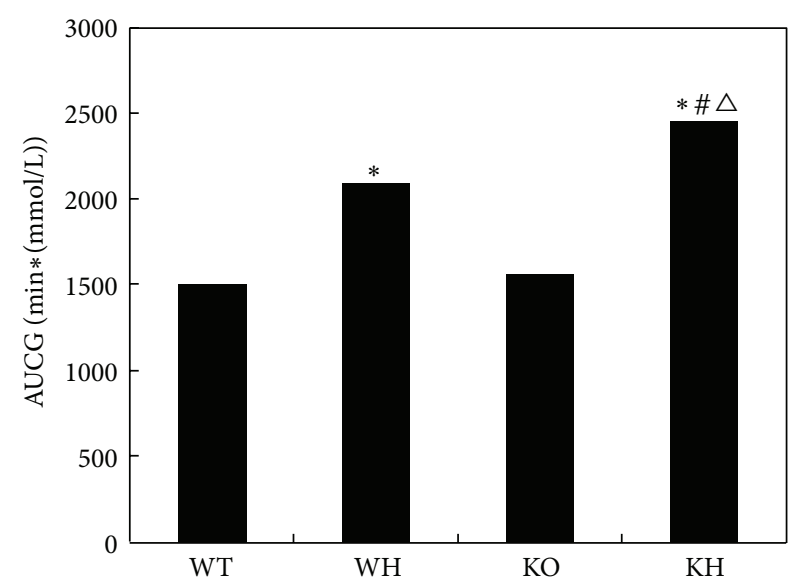

(b)

FIgURE 2: The curve of glucose tolerance test. (a) Glucose curve; (b) area under curve of glucose (AUCG). WT: wildtype mice; WH: wildtype mice with high-fat diet; KO: ACE2 knockout mice; KH: ACE2 KO mice with high-fat diet, versus $\mathrm{WT}^{*} P<0.05$, versus KO ${ }^{*} P<0.05$, and versus $\mathrm{WH}^{\triangle} \mathrm{P}<0.05$.

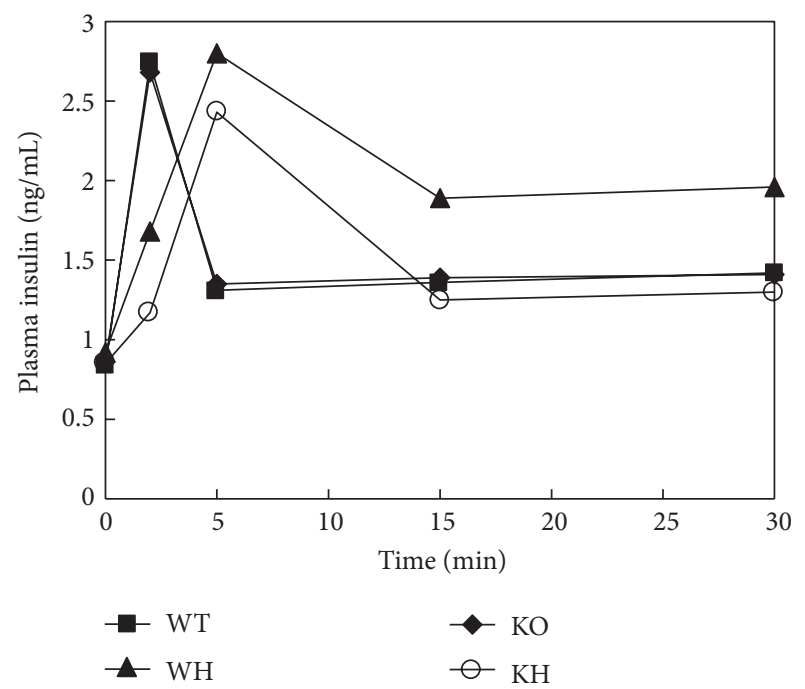

FIGURE 3: The curve of insulin releasing test. WT: wildtype mice; WH: wildtype mice with high-fat diet; KO: ACE2 knockout mice; $\mathrm{KH}$ : ACE2 KO mice with high-fat diet.

demonstrated enhanced MVD in WH group than WT group $(P<0.05)$, which suggested higher islet vascularization after high-fat diet. However, the $\mathrm{KH}$ mice did not show an increased MVD in islet when compared with $\mathrm{KO}$ mice. Moreover, there was reduced MVD in islet from KH group when compared with $\mathrm{WH}$ group $(P<0.05)$. These findings indicated that loss of ACE2 decreases the compensation of islet vascularization in response to high-fat diet (Figure 5, Table 2).

3.6. VRC Intraislets. The VRC was similar between the WT and $\mathrm{KO}$ group. In comparison with WT group, VRC of WH

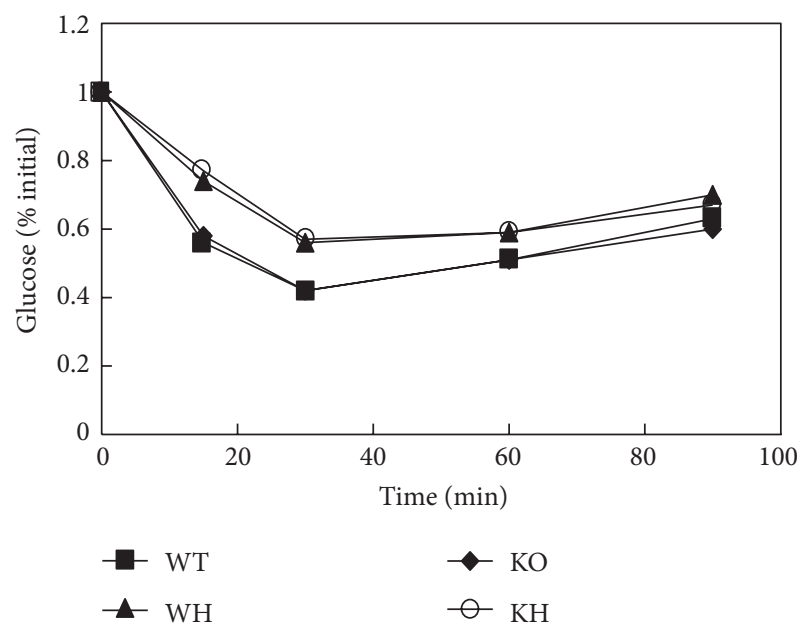

FIGURE 4: The curve of insulin tolerance test. WT: wildtype mice; WH: wildtype mice with high-fat diet; KO: ACE2 knockout mice; $\mathrm{KH}$ : ACE2 KO mice with high-fat diet.

group was increased $(P<0.05)$. However, there was no difference of VRC between $\mathrm{KO}$ group and $\mathrm{KH}$ group. The VRC in $\mathrm{KH}$ group was decreased when compared with $\mathrm{WH}$ group $(P<0.05)$ (Figure 5, Table 2$)$.

\section{Discussion}

The RAS has an important role in the endocrine pancreas [7]. Many projects have focused on the effects of ACE-Ang II-AT1 axis. A homolog of ACE, ACE2 cleaves the terminal phenylalanine residue from Ang II to synthesis Ang (1-7), thereby functioning as a negative regulator of the RAS. In this 
TABLE 2: Data of immunohistochemistry (mean $\pm \mathrm{SD}$ ).

\begin{tabular}{lcccc}
\hline & WT & WH & KO & KH \\
\hline Insulin relative concentration & $-4.00 \pm 0.05$ & $-4.63 \pm 0.06^{*}$ & $-4.10 \pm 0.04$ & $-5.07 \pm 0.06^{* \# \Delta}$ \\
Insulin-positive cell density & $2.35 \pm 0.02$ & $2.30 \pm 0.01$ & $2.32 \pm 0.02$ & $2.28 \pm 0.01$ \\
Microvascular density & $13.52 \pm 0.48$ & $17.09 \pm 0.52^{*}$ & $11.87 \pm 0.45$ & $7.89 \pm 0.46^{* \# \Delta}$ \\
Relative content of VEGF & $-4.67 \pm 0.11$ & $-3.99 \pm 0.05^{*}$ & $-4.91 \pm 0.04$ & $-5.11 \pm 0.04^{* \# \Delta}$ \\
\hline
\end{tabular}

WT: wild type mice; WH: wild type mice with high-fat diet; KO: ACE2 knockout mice; KH: ACE2 knockout mice with high-fat diet. VEGF: vascular endothelial growth factor, versus $\mathrm{WT}^{*} P<0.05$, versus $\mathrm{KO}^{\#} P<0.05$, and versus $\mathrm{WH}^{\triangle} P<0.05$.
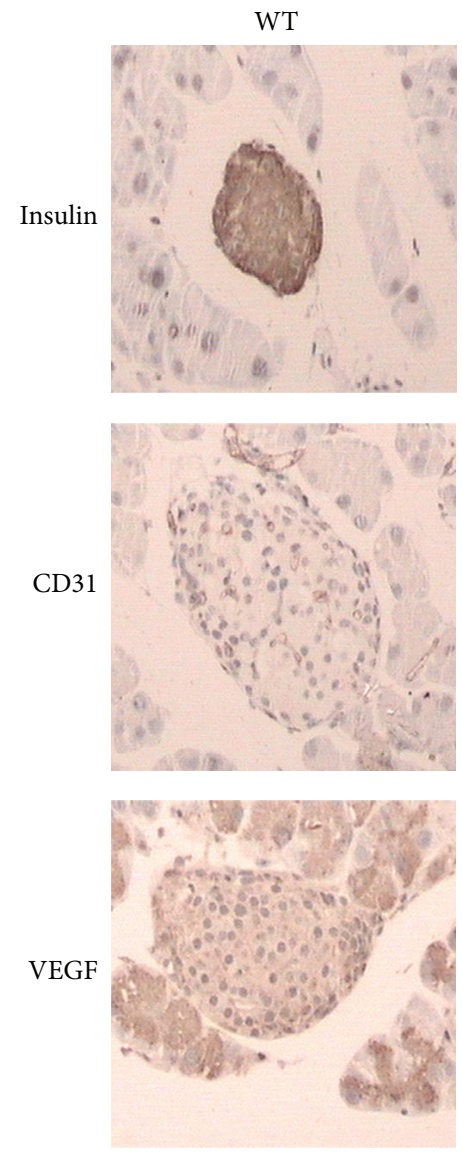
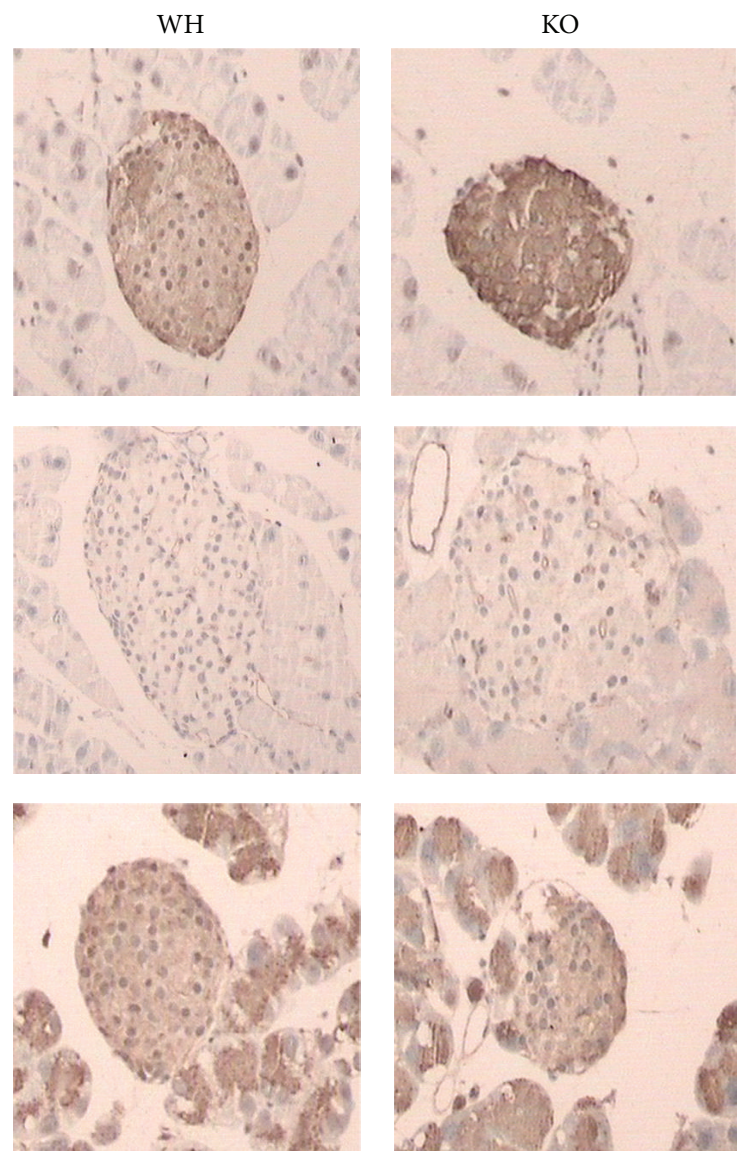
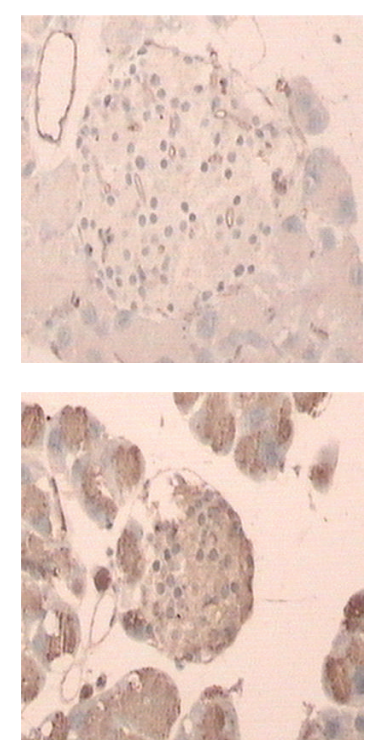
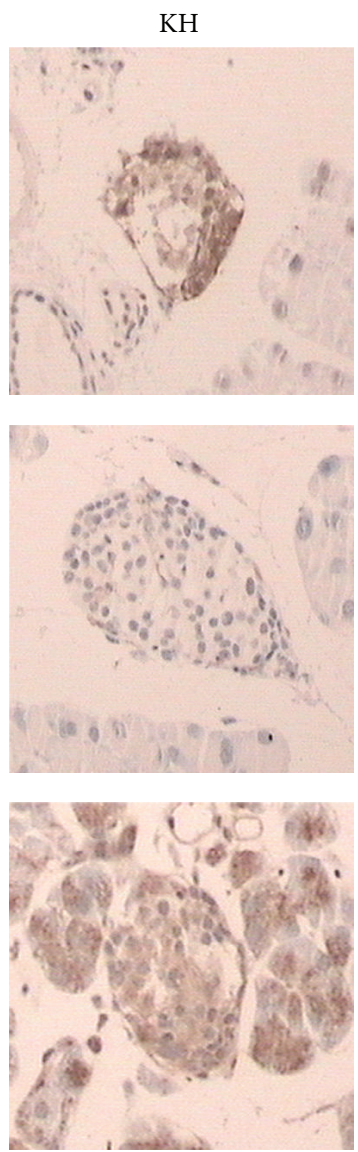

FIGURE 5: Immunohistochemistry. WT: wildtype mice; WH: wildtype mice with high-fat diet; KO: ACE2 knockout mice; KH: ACE2 KO mice with high-fat diet. VEGF: vascular endothelial growth factor.

current study, we demonstrated that high-fat-diet-induced ACE2 KO mice exhibit a progressive impairment of glucose tolerance and a reduction of insulin secretion in response to glucose, compared with WT mice. In the high-fat-induced ACE2 KO mice, loss of ACE2 triggered a greater decrease in islet $\beta$ cell and islet MVD. Our study supported a pivotal role of ACE2 in the regulation of $\beta$ cell function and islet vasculature.

As the major bioactive component of the classical RAS, Ang II mainly interacts with the AT1 receptor to exert various actions including vasoconstriction, activation of proinflammation, fibrosis and oxidative stress. ACE2 is a monocarboxypeptidase cleaving Ang II to form Ang (1-7). As a class of G-protein-coupled receptor, Mas have been identified as a receptor of Ang (1-7). Ang (1-7) interacting with Mas elicits protective actions such as vasodilatation and nitric oxide generation that counterbalance those of Ang II [6]. The ACE2-Ang (1-7)-Mas axis may act as a negative modulator of the classical RAS effects [24]. There is emerging evidence for a functional ACE2-Ang (1-7)-Mas axis which is more than regulation of cardiovascular system [25].

The association of the classic RAS with the endocrine system has been identified in diabetes and metabolic syndrome [26]. A large number of clinical trials have indicated that inhibitors of the RAS, such as ACEI and ARB, reduce the incidence of new-onset T2DM in high-risk individuals and protect against the development of T2DM $[19,20]$. Furthermore, inhibition of RAS has been also reported to exert protective action to glucose homeostasis in animal models of T2DM [27]. On the other hand, ACE2 may be 
a potential protective enzyme for glucose homeostasis [9], but the role of ACE2 in the regulation of islet function is incompletely understood. In the present study, there were on differences in fasting blood glucose and glucose tolerance in male ACE2 KO mice with standard chow diet compared with corresponding WT mice. This observation was consistent with a previous study by Wong et al. [22], who found that glycemic control was not worsened by the absence of ACE2 in the Akita diabetic mice. Therefore, we supposed that ACE2 ablation does not seem to be sufficient to alter glucose status in the condition of regular chow diet. However, an interesting and important finding of the current study was that the consequence of ACE2 absence became apparent after highfat diet. Under the condition of high-fat diet, there were much more reductions of the first-stage insulin secretion and glucose tolerance in $\mathrm{KH}$ mice compared with WH mice. Not surprisingly, these changes were linked to decreased $\beta$ cell mass. It is noteworthy that this finding was partly supported by the report of gene therapy in obese diabetic mice. Bindom et al. [28] found that ACE2 gene therapy was able to improve fasting blood glucose and glucose tolerance in obese diabetic mice. Consistent with the improvement of glycemic control, ACE2 gene therapy leaded to enhanced first-stage insulin secretion and reduced $\beta$ cell apoptosis. Our current study for the first time provided evidence that ACE2 absence leads to more severe effects in $\beta$ cell dysfunction in obese mice with high-fat diet. Taken together, it is hypothesized that loss of ACE2 renders the pancreatic islet more susceptible to the pathological actions of obesity induced by high-fat diet.

It is known that islet vascularization is essential for the islet to respond to fluctuations in blood glucose and modulate insulin release as needed. Defects in the vascularization of pancreatic islets can lead to deleterious effects on glucose tolerance and islet insulin secretion. In our study, an increased islet vascularization in high-fat diet WT mice was observed compared with chow diet WT mice, which represented a compensatory response to support higher vascularization during $\beta$ cell expansion. In contrast, no increased islet vascularization was observed in $\mathrm{KH}$ mice compared with KO mice. Furthermore, $\mathrm{KH}$ mice exhibited defects of islet vascularization compared with $\mathrm{WH}$ mice. These findings suggested that deletion of ACE2 reduces the compensation of islet vascularization in response to high-fat diet. There is an intimate connection between islet beta cell and islet vascularization. On the one hand, islet vascularization has been regarded as playing a crucial role in maintaining beta cell survival and function. On the other hand, beta cells secrete several factors such as VEGF to promote vascular development. VEGF, which is highly expressed in islet, has been identified as an important factor to be involved in the regulation of islet vascularization $[23,29]$. Vascular endothelial cells migrate to the source of VEGF-A and proliferate and form blood vessels in response to VEGF [30]. Previous studies [31, 32] suggested that mice with specific deletion of the VEGF in $\beta$ cells had reduced islet vascularization and impaired glucose tolerance. These findings were in line with observations in the transplanted pancreatic islets. It has been reported that reduction of VEGF-A by $\beta$ cells not only reduces the number of intraislet endothelial cells participating in graft vessel formation but also limits recruitment of host endothelial cell and their invasion into the graft $[31,33]$. In our study, we found an increase of islet VEGF in $\mathrm{WH}$ mice, which was consistent with the enhancement of islet vascularization after high-fat diet. However, with the deletion of ACE2, this compensatory increase of VEGF in islet was disappeared after high-fat diet. This was parallel to the islet $\beta$ cell impairment and islet vascularization reduction. With the defects of the islet vascularization, the beta cell was impaired. The deteriorated beta cell might lead to a reduction of VEGF expression which in turn accelerates the loss of vascularization. Taken together, we speculated that under the condition of high-fat diet, ACE2 might play a critical role in the glucose intolerance through impairment of islet vascularization.

ACE2 not only functions as a carboxypeptidase, cleaving a single residue from Ang I, generating Ang1-9, and a single residue from Ang II to generate Ang 1-7 but also cleaves several other biological peptides such as apelin. ACE2 hydrolyzes apelin with high catalytic efficiency [34]. Apelin is the endogenous ligand of the G-protein coupled apj receptor. The effects of apelin on the pancreas focused on the regulation of insulin secretion [35]. Winzell et al. [36] reported that apelin-36 inhibited the glucose-stimulated insulin secretion in obese insulin resistant high-fat fed mice. Moreover, apelin-13 was shown to inhibit insulin secretion stimulated by high glucose concentrations in INS-1 cells [37]. Accordingly, we hypothesized that with the deletion of ACE2, the hydrolyzation of apelin is inhibited, which leads to the inhibition of insulin secretion.

Peripheral Insulin sensitivity has long been regarded as playing a crucial role in glucose homeostasis. However, the effect of ACE2 on insulin sensitivity is unclear. In this study, we took insulin tolerance test to evaluate peripheral insulin sensitivity. We found no significant difference of peripheral insulin sensitivity between $\mathrm{KO}$ mice and WT mice. The peripheral insulin sensitivity between $\mathrm{WH}$ mice and $\mathrm{KH}$ mice was similar too. So, we concluded that loss of ACE2 has no effects on peripheral insulin sensitivity. More accurate measures such as hyperinsulinemic euglycemic clamp should be needed in further studies. It should be noted that there was no difference of the body weight between WT and ACE2 $\mathrm{KO}$ mice neither with chow diet nor with high-fat diet. Accordingly, we speculated that ACE2 was not involved in the regulation of body weight.

In summary, the results obtained in this study show that loss of ACE2 in mice with long-term high-fat diet leads to impairment of glucose tolerance and insulin secretion. The primary mechanisms involved in these effects appear to include a reduction in islet $\beta$ cell and islet vascularization. Our data suggested that the absence of ACE2 weakened the adaptive changes of islet $\beta$ cell function and islet vascularization due to high-fat diet. ACE2 seems to be a potential therapeutic target in the treatment of islet dysfunction induced by high-fat diet. 


\section{Acknowledgment}

This project was supported by the National Natural Science Foundation of China (no. 81070615).

\section{References}

[1] J. R. A. Skipworth, G. Szabadkai, S. W. M. Olde Damink, P. S. Leung, S. E. Humphries, and H. E. Montgomery, "Review article: pancreatic renin-angiotensin systems in health and disease," Alimentary Pharmacology \& Therapeutics, vol. 34, no. 8, pp. 840-852, 2011.

[2] S. R. Tipnis, N. M. Hooper, R. Hyde, E. Karran, G. Christie, and A. J. Turner, "A human homolog of angiotensin-converting enzyme: cloning and functional expression as a captoprilinsensitive carboxypeptidase," Journal of Biological Chemistry, vol. 275, no. 43, pp. 33238-33243, 2000.

[3] M. Donoghue, F. Hsieh, E. Baronas et al., "A novel angiotensinconverting enzyme-related carboxypeptidase (ACE2) converts angiotensin I to angiotensin 1-9," Circulation Research, vol. 87, no. 5, pp. el-e9, 2000.

[4] G. I. Rice, D. A. Thomas, P. J. Grant, A. J. Turner, and N. M. Hooper, "Evaluation of angiotensin-converting enzyme (ACE), its homologue ACE2 and neprilysin in angiotensin peptide metabolism," Biochemical Journal, vol. 383, no. 1, pp. 45-51, 2004.

[5] C. M. Ferrario, "ACE2: more of Ang-(1-7) or less Ang II?" Current Opinion in Nephrology and Hypertension, vol. 20, no. 1, pp. 1-6, 2011.

[6] R. A. S. Santos, A. J. Ferreira, and A. C. Simões E Silva, "Recent advances in the angiotensin-converting enzyme 2 -angiotensin(1-7)-Mas axis," Experimental Physiology, vol. 93, no. 5, pp. 519-527, 2008.

[7] Q. Cheng and P. S. Leung, "An update on the islet renin-angiotensin system," Peptides, vol. 32, no. 5, pp. 1087-1095, 2011.

[8] P. S. Leung, "The physiology of a local renin-angiotensin system in the pancreas," Journal of Physiology, vol. 580, no. 1, pp. 31-37, 2007.

[9] D. Batlle, M. J. Soler, and M. Ye, "ACE2 and diabetes: ACE of ACEs?” Diabetes, vol. 59, no. 12, pp. 2994-2996, 2010.

[10] M. C. Chappell, A. Millsted, D. I. Diz, K. B. Brosnihan, and C. M. Ferrario, "Evidence for an intrinsic angiotensin system in the canine pancreas," Journal of Hypertension, vol. 9, no. 8, pp. 751-759, 1991.

[11] T. Lau, P. O. Carlsson, and P. S. Leung, "Evidence for a local angiotensin-generating system and dose-dependent inhibition of glucose-stimulated insulin release by angiotensin II in isolated pancreatic islets," Diabetologia, vol. 47, no. 2, pp. 240-248, 2004.

[12] K. Y. Lam and P. S. Leung, "Regulation and expression of a renin-angiotensin system in human pancreas and pancreatic endocrine tumours," European Journal of Endocrinology, vol. 146, no. 4, pp. 567-572, 2002.

[13] C. Tikellis, M. E. Cooper, and M. C. Thomas, "Role of the reninangiotensin system in the endocrine pancreas: implications for the development of diabetes," International Journal of Biochemistry and Cell Biology, vol. 38, no. 5-6, pp. 737-751, 2006.

[14] S. M. Bindom and E. Lazartigues, "The sweeter side of ACE2: physiological evidence for a role in diabetes," Molecular and Cellular Endocrinology, vol. 302, no. 2, pp. 193-202, 2009.

[15] P. S. Leung and P. O. Carlsson, "Pancreatic islet renin angiotensin system: its novel roles in islet function and in diabetes mellitus," Pancreas, vol. 30, no. 4, pp. 293-298, 2005.
[16] P. S. Leung, "Mechanisms of protective effects induced by blockade of the renin-angiotensin system: novel role of the pancreatic islet angiotensin-generating system in Type 2 diabetes," Diabetic Medicine, vol. 24, no. 2, pp. 110-116, 2007.

[17] C. Tikellis, P. J. Wookey, R. Candido, S. Andrikopoulos, M. C. Thomas, and M. E. Cooper, "Improved islet morphology after blockade of the renin-angiotensin system in the ZDF rat," Diabetes, vol. 53, no. 4, pp. 989-997, 2004.

[18] L. Yuan, X. Li, J. Li, H. 1. Li, and S. S. Cheng, "Effects of reninangiotensin system blockade on the islet morphology and function in rats with long-term high-fat diet," Acta Diabetologica. In press.

[19] A. J. Scheen, "Renin-angiotensin system inhibition prevents type 2 diabetes mellitus-part 1: a meta-analysis of randomised clinical trials," Diabetes and Metabolism, vol. 30, no. 6, pp. 487496, 2004.

[20] E. L. Gillespie, C. M. White, M. Kardas, M. Lindberg, and C. I. Coleman, "The impact of ACE inhibitors or angiotensin II type 1 receptor blockers on the development of new-onset type 2 diabetes," Diabetes Care, vol. 28, no. 9, pp. 2261-2266, 2005.

[21] P. Vejakama, A. Thakkinstian, D. Lertrattananon, A. Ingsathit, C. Ngarmukos, and J. Attia, "Reno-protective effects of reninangiotensin system blockade in type 2 diabetic patients: a systematic review and network meta-analysis," Diabetologia, vol. 55, no. 3, pp. 566-578, 2011.

[22] D. W. Wong, G. Y. Oudit, H. Reich et al., "Loss of Angiotensinconverting enzyme-2 (Ace2) accelerates diabetic kidney injury," American Journal of Pathology, vol. 171, no. 2, pp. 438-451, 2007.

[23] E. M. Akirav, M. T. Baquero, L. W. Opare-Addo et al., "Glucose and inflammation control islet vascular density and $\beta$-cell function in NOD mice control of islet vasculature and vascular endothelial growth factor by glucose," Diabetes, vol. 60, no. 3, pp. 876-883, 2011.

[24] M. Iwai and M. Horiuchi, "Devil and angel in the renin-angiotensin system: ACE-angiotensin II-AT1 receptor axis vs. ACE2angiotensin-(1-7)-Mas receptor axis," Hypertension Research, vol. 32, no. 7, pp. 533-536, 2009.

[25] M. C. Chappell, "Emerging evidence for a functional angiotensin-converting enzyme 2-angiotensin-(1-7)-Mas receptor axis: more than regulation of blood pressure?" Hypertension, vol. 50, no. 4, pp. 596-599, 2007.

[26] K. Putnam, R. Shoemaker, F. Yiannikouris, and L. Cassis, “The renin-angiotensin system: a target of and contributor to dyslipidemias, altered glucose homeostasis, and hypertension of the metabolic syndrome," American Journal of Physiology, vol. 15, no. 302, pp. H1219-H1230, 2012.

[27] V. Souza-Mello, B. M. Gregório, F. S. Cardoso-De-Lemos, L. De Carvalho, M. B. Aguila, and C. A. Mandarim-De-Lacerda, "Comparative effects of telmisartan, sitagliptin and metformin alone or in combination on obesity, insulin resistance, and liver and pancreas remodelling in C57BL/6 mice fed on a very highfat diet," Clinical Science, vol. 119, no. 6, pp. 239-250, 2010.

[28] S. M. Bindom, C. P. Hans, H. Xia, A. H. Boulares, and E. Lazartigues, "Angiotensin I-converting enzyme type 2 (ACE2) gene therapy improves glycemic control in diabetic mice," Diabetes, vol. 59, no. 10, pp. 2540-2548, 2010.

[29] J. Agudo, E. Ayuso, V. Jimenez et al., "Vascular endothelial growth factor-mediated islet hypervascularization and inflammation contribute to progressive reduction of $\beta$-cell mass," Diabetes, vol. 61, no. 11, pp. 2851-2861, 2012.

[30] O. Cleaver and Y. Dor, "Vascular instruction of pancreas development," Development, vol. 139, no. 16, pp. 2833-2843, 2012. 
[31] M. Brissova, A. Shostak, M. Shiota et al., "Pancreatic islet production of vascular endothelial growth factor-A is essential for islet vascularization, revascularization, and function," Diabetes, vol. 55, no. 11, pp. 2974-2985, 2006.

[32] N. Jabs, L. Franklin, M. B. Brenner et al., "Reduced insulin secretion and content in VEGF-A deficient mouse pancreatic islets," Experimental and Clinical Endocrinology and Diabetes, vol. 116, no. 1, pp. S46-S49, 2008.

[33] M. Brissova, M. Fowler, P. Wiebe et al., "Intraislet endothelial cells contribute to revascularization of transplanted pancreatic islets," Diabetes, vol. 53, no. 5, pp. 1318-1325, 2004.

[34] C. Vickers, P. Hales, V. Kaushik et al., "Hydrolysis of biological peptides by human angiotensin-converting enzyme-related carboxypeptidase," Journal of Biological Chemistry, vol. 277, no. 17, pp. 14838-14843, 2002.

[35] I. Castan-laurell, C. Dray, C. Knauf, O. Kunduzova, and P. Valet, "Apelin, a promising target for type 2 diabetes treatment?" Trends in Endocrinology \& Metabolism, vol. 23, no. 5, pp. 234241, 2012.

[36] M. S. Winzell, C. Magnusson, and B. Ahrén, “The apj receptor is expressed in pancreatic islets and its ligand, apelin, inhibits insulin secretion in mice," Regulatory Peptides, vol. 131, no. 1-3, pp. 12-17, 2005.

[37] L. Guo, Q. Li, W. Wang et al., "Apelin inhibits insulin secretion in pancreatic $\beta$-cells by activation of PI3-kinase-phosphodiesterase 3B," Endocrine Research, vol. 34, no. 4, pp. 142-154, 2009. 


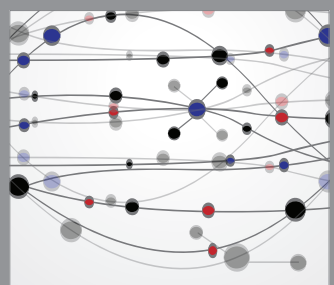

The Scientific World Journal
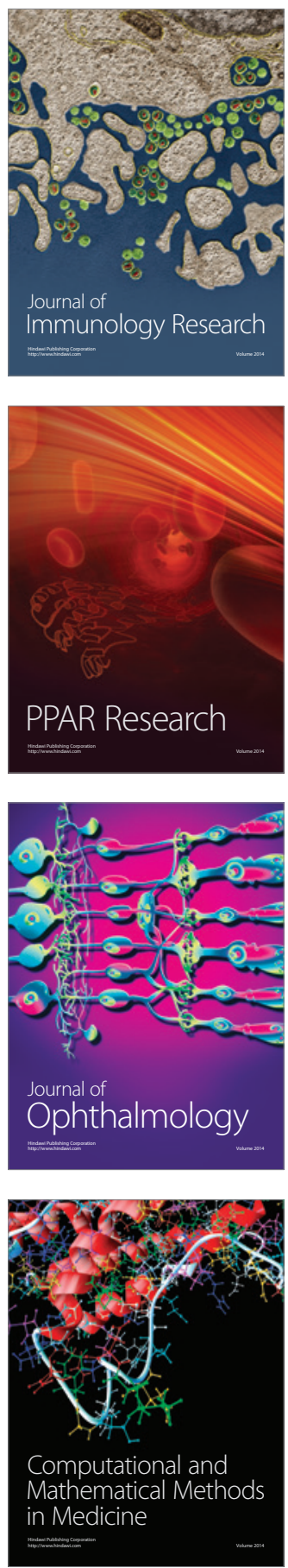

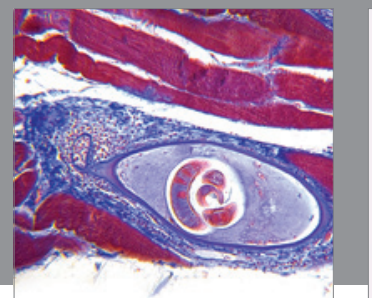

Gastroenterology

Research and Practice
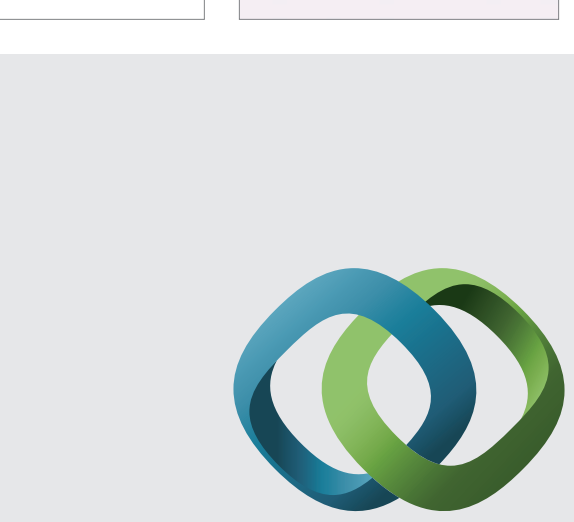

\section{Hindawi}

Submit your manuscripts at

http://www.hindawi.com
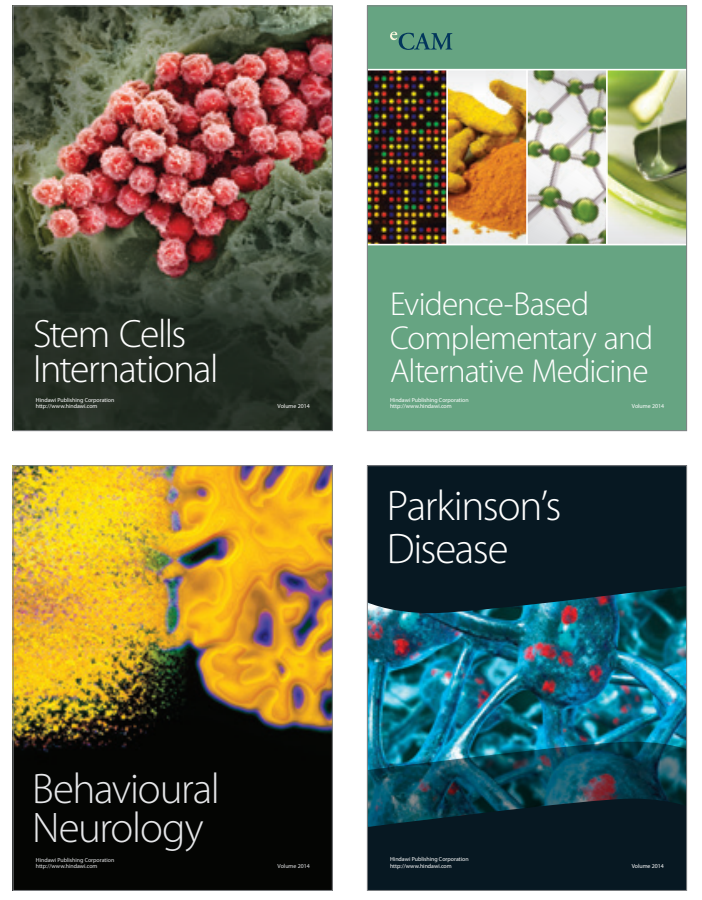
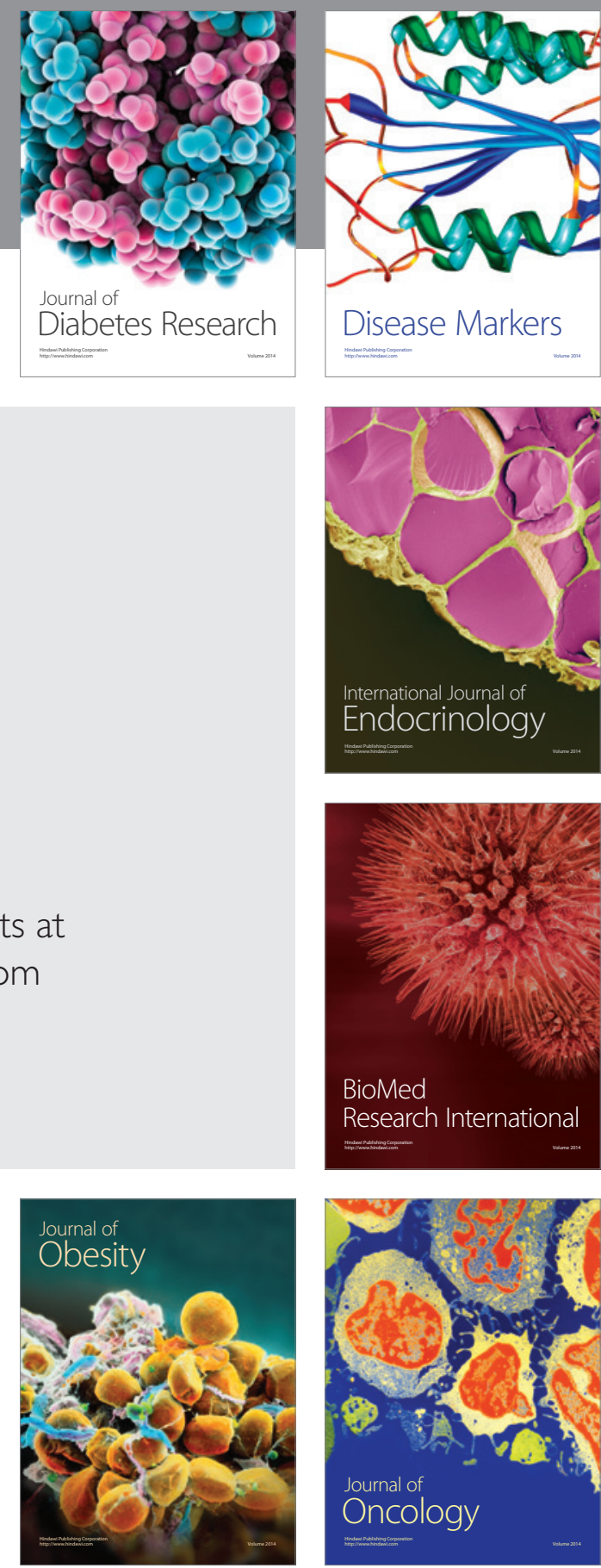

Disease Markers
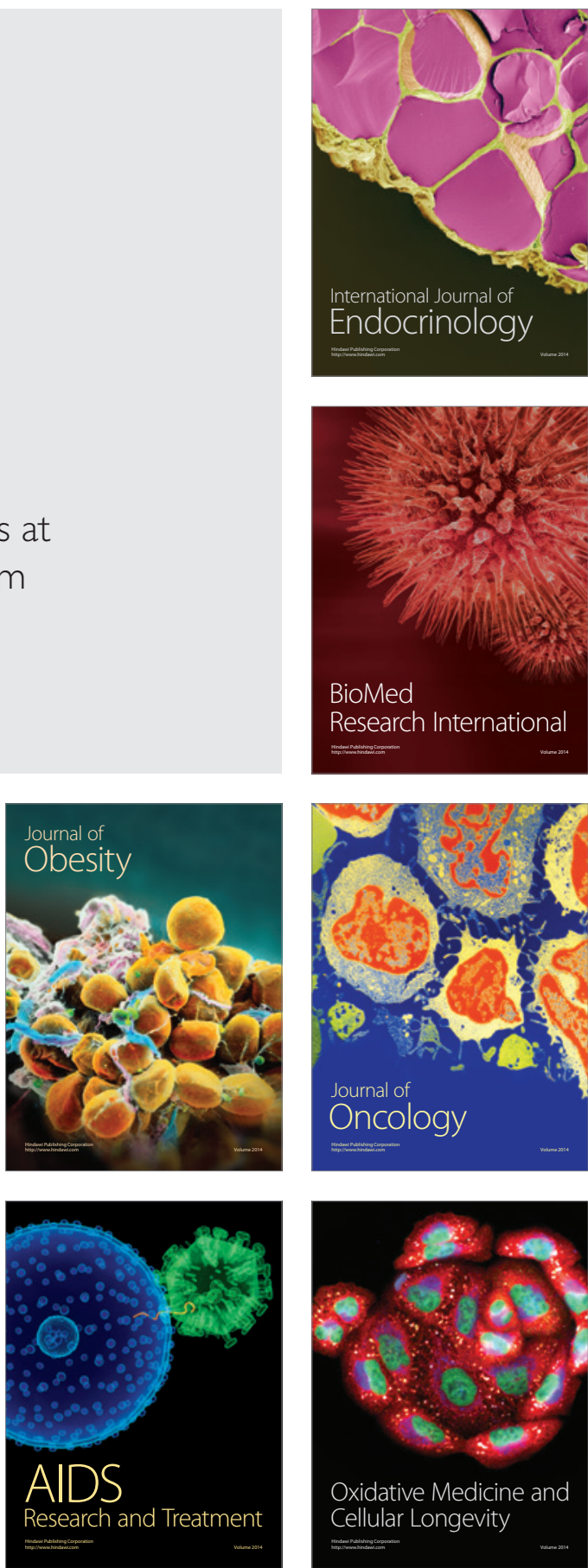